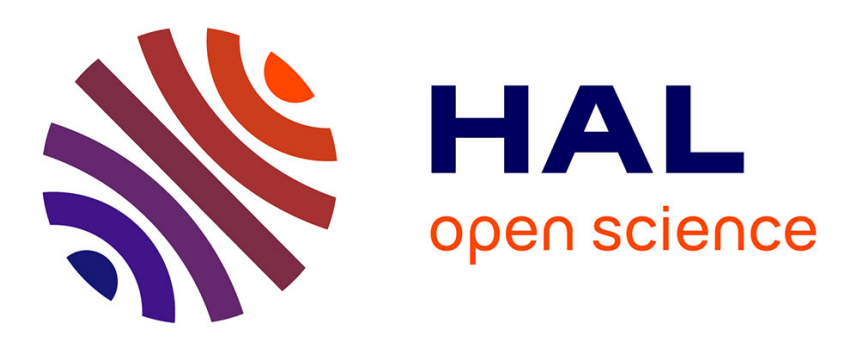

\title{
Emploi de fronts d'ondes quasi limités dans certains problèmes de diffraction. des ondes électromagnétiques. Application à la lentille cylindrique \\ P. Facq, W. Tabbara
}

\section{- To cite this version: \\ P. Facq, W. Tabbara. Emploi de fronts d'ondes quasi limités dans certains problèmes de diffraction. des ondes électromagnétiques. Application à la lentille cylindrique. Revue de Physique Appliquée, 1971, 6 (2), pp.191-194. 10.1051/rphysap:0197100602019100 . jpa-00243522}

HAL Id: jpa-00243522

https://hal.science/jpa-00243522

Submitted on 1 Jan 1971

HAL is a multi-disciplinary open access archive for the deposit and dissemination of scientific research documents, whether they are published or not. The documents may come from teaching and research institutions in France or abroad, or from public or private research centers.
L'archive ouverte pluridisciplinaire HAL, est destinée au dépôt et à la diffusion de documents scientifiques de niveau recherche, publiés ou non, émanant des établissements d'enseignement et de recherche français ou étrangers, des laboratoires publics ou privés. 


\title{
EMPLOI DE FRONTS D'ONDES QUASI LIMITÉS DANS CERTAINS PROBLËMES DE DIFFRACTION. DES ONDES ÉLECTROMAGNÉTIQUES APPLICATION A LA LENTILLE CYLINDRIQUE
}

\author{
P. FACQ \\ Laboratoire d'Optique, Faculté des Sciences, 87, Limoges \\ W. TABBARA \\ Institut d'Optique, Paris
}

\begin{abstract}
Résumé. - Nous proposons des modèles de sources destinés à produire les fronts d'onde incidents limités dont l'usage est fréquent dans les expériences de diffraction.

Le champ rayonné par un ruban cohérent a été utilisé comme champ incident dans le traitement numérique du problème de la diffraction d'une onde quasi limitée par une lentille cylindrique.
\end{abstract}

\begin{abstract}
In many diffraction and scattering experiments the incident wavefronts are limited. We propose a model of source that can be appropriate to simulate this limitation.

The field radiated by a coherent ribbon source has been used as an incident field in the problem of diffraction of a quasi limited wavefront by a cylindrical lens.
\end{abstract}

Introduction. - Nous traitons des problèmes cylindriques. Dans l'espace rapporté à un trièdre trirectangle $O x y z$, les constantes des milieux, les frontières des obstacles, les champs sont indépendants de l'une des coordonnées, $y$ par exemple. La discussion est restreinte aux polarisations $\mathrm{T}$. $\mathrm{E}$. (E parallèle à $o y$ ) ou T. M. (H parallèle à oy). On se place dans le cas de la cohérence temporelle parfaite (facteur temporel $\left.\mathrm{e}^{-i \omega t}\right)$. Dans ces conditions le champ électromagnétique est décrit dans tout l'espace par un scalaire $U(x, z)$ qui est, selon le cas, l'amplitude complexe de $\mathbf{E}$ (cas T. E.) ou de $\mathbf{H}$ (cas T. M.).

Le champ incident dans les problèmes théoriques de diffraction à deux dimensions est habituellement une onde plane infinie ou une onde cylindrique. Divers essais d'utilisation de fronts d'onde limités ont provoqué les critiques des théoriciens [1]. Considérons par exemple le modèle simple d'un faisceau cylindrique caractérisé par une amplitude complexe

$$
U(x, z)=\operatorname{rect}(x / 2 a) \mathrm{e}^{i k z}
$$

dont le module est égal à l'unité pour $|x|<a$ et nul pour $|x|>a$. Un tel champ ne satisfait pas les équations de Maxwell dans l'espace libre car :

$\left(\Delta+k^{2}\right) U(x, z)=\left[\delta^{\prime}(x+a)-\delta^{\prime}(x-a)\right] \mathrm{e}^{i k z}$.

Cette équation montre que le champ $U$ ne peut garder, au cours de sa propagation, la forme décrite par (1) qu'en présence de sources dipôles réparties sur les plans $x=a$ et $x=-a$.
Or, dans de nombreux problèmes, on a besoin de représenter un faisceau dirigé se propageant dans l'espace vide de sources.

Rayonnement des sources ruban cohérentes. Considérons les sources dont les densités sont données par

$$
\begin{aligned}
& \rho_{E}(x, z)=2 k f(x) \operatorname{rect}(x / D) \delta(z) \\
& \rho_{M}(x, z)=2 g(x) \operatorname{rect}(x / D) \delta^{\prime}(z) .
\end{aligned}
$$

Ces densités correspondent à des répartitions de filaments unipolaires (3) ou dipolaires (4) parallèles à oy et localisés sur une bande - un ruban - du plan xoy. $f(x)$ et $g(x)$ sont des scalaires complexes.

Si $U$ satisfait les conditions de rayonnement à l'infini de Sommerfeld (régularité à l'infini et condition d'onde sortante) l'application de la deuxième formule de Green à $U$ et à la fonction de Green cylindrique $G$ conduit aux expressions suivantes pour les champs rayonnés $\operatorname{par} \rho_{E}$ et $\rho_{M}[2]$

$U_{E}\left(x^{\prime}, z^{\prime}\right)=2 k \int_{-D / 2}^{D / 2} f(x) G\left(x^{\prime}-x, z^{\prime}\right) \mathrm{d} x$

$U_{M}\left(x^{\prime}, z^{\prime}\right)=-2 \int_{-D / 2}^{D / 2} g(x) \partial_{z} G\left(x^{\prime}-x, z^{\prime}\right) \mathrm{d} x$

avec

$G\left(x^{\prime}-x, z^{\prime}-z\right)=\frac{i}{4} H_{0}^{(1)}\left[k \sqrt{\left(x^{\prime}-x\right)^{2}+\left(z^{\prime}-z\right)^{2}}\right]$ 
$H_{0}^{(1)}$ étant la fonction de Hankel de première espèce et d'ordre 0 .

A titre d'exemple le champ donné par la relation (5) a été évalué numériquement pour $D=100 \lambda$ et $f(x)=-i$. La figure 1 montre $|U|$ dans divers plans
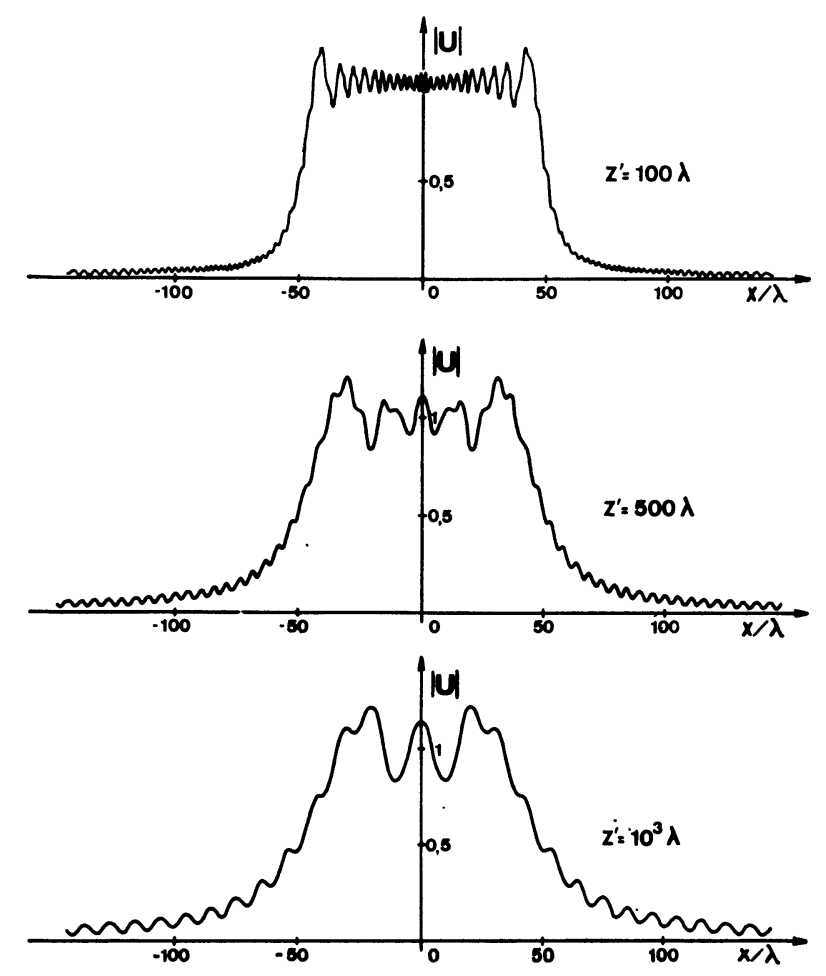

Fig. 1. - Coupes du faisceau rayonné par une source ruban unipolaire de largeur $D=100 \lambda$.

parallèles à la source. Dans la région dite de Fresnel $\left(z^{\prime}<D^{2} / \lambda\right),|U|$ oscille autour de l'unité dans une bande de largeur $D$ et tend vers 0 en dehors. C'est pour cette raison que l'on parlera de fronts d'onde «quasi limités $»$.

Relations avec le problème de la diffraction par une fente. - Les fronts d'onde quasi limités générés par les sources ruban ont une parenté étroite avec le champ diffracté par une fente dans un écran conducteur mince recevant une onde d'amplitude complexe $U_{i}(x, z)$.

Si dans les expressions des champs (5) et (6) nous faisons

$$
\begin{aligned}
& f(x)=-\frac{1}{k} \partial_{z} U_{i}(x, 0) \\
& g(x)=-U_{i}(x, 0)
\end{aligned}
$$

nous obtenons, pour le demi-espace côté émergent, les relations approchées de Rayleigh-Sommerfeld (*).

\footnotetext{
(*) L'intégrale $\int_{-D / 2}^{D / 2}$ portant sur l'ouverture est notée symboliquement $\int_{0}$.
}

Cas T. E.

$U_{E}\left(x^{\prime}, z^{\prime}\right)=-2 \int_{0} \partial_{z}^{\mathbf{l}} U_{i}(x, 0) G\left(x^{\prime}-x, z^{\prime}\right) \mathrm{d} x ; z^{\prime}>0$

Cas T. M.

$U_{M}\left(x^{\prime}, z^{\prime}\right)=2 \int_{0} U_{i}(x, 0) \partial_{z} G\left(x^{\prime}-x, z^{\prime}\right) \mathrm{d} x ; \quad z^{\prime}>0$

citées par Bouwkamp [3] et qui s'obtiennent également comme solution d'un problème de sauts sur l'écran [4].

Nous avons testé numériquement la validité de cette approximation par comparaison avec le champ diffracté $U_{d}$ fourni par la solution rigoureuse du même problème, dont nous rappelons les résultats pour le demi-espace côté émergent.

Cas T. E.

$$
\begin{gathered}
U_{d}\left(x^{\prime}, z^{\prime}\right)=2 \int_{0} U_{d}(x, 0) \partial_{z} G\left(x^{\prime}-x, z^{\prime}\right) \mathrm{d} x ; z^{\prime}>0 \\
2 P F \int_{0} U_{d}(x, 0) \partial_{z^{\prime}} \partial_{z} G\left(x^{\prime}-x, 0\right) \mathrm{d} x=\partial_{z^{\prime}} U_{i}\left(x^{\prime}, 0\right) ; \\
\left|x^{\prime}\right|<\frac{D}{2} .
\end{gathered}
$$

Cas T. M.

$U_{d}\left(x^{\prime}, z^{\prime}\right)=-2 \int_{0} \partial_{z} U_{d}(x, 0) G\left(x^{\prime}-x, z^{\prime}\right) \mathrm{d} x ; z^{\prime}>0$

$$
\begin{gathered}
-2 \int_{0} \partial_{z} U_{d}(x, 0) G\left(x^{\prime}-x, 0\right) \mathrm{d} x=U_{i}\left(x^{\prime}, 0\right) ; \\
\left|x^{\prime}\right|<\frac{D}{2} .
\end{gathered}
$$

L'intégrale figurant dans l'équation (13) est divergente mais Filippi et Dumery [5] ont montré que l'expression était utilisable à condition d'envisager l'intégration au sens des parties finies.

Nous avons effectué la comparaison sur les champs dans l'ouverture illuminée sous l'incidence $\theta$ par une onde plane d'amplitude unité. La figure 2 montre les résultats de cette comparaison pour le cas T. E.

Sur les courbes décrivant $|U|$, le tracé en trait plein correspond à $U_{E}$ (équation (10)) ; les cercles se rapportent au calcul de $U_{d}$ (équation intégrale (13)) par une variante de la méthode des moments décrite par Harrington [6] sous le nom de "point matching ». Nous avons utilisé 50 points d'échantillonnage pour $D=4 \lambda$ et 100 points pour $D=10 \lambda . \Delta \varphi$ représente l'écart de phase entre $U_{E}$ et $U_{d}$.

En ce qui concerne les champs hors de l'ouverture, les formules approchées et rigoureuses se présentent sous des formes apparemment très différentes. Pour le même cas T. E., la solution exacte (12) correspond au 
rayonnement de dipôles fictifs localisés dans l'ouverture, alors que la relation approchée (10) fait intervenir des sources unipôles. Cependant pour $D=4 \lambda$, en incidence normale, les diagrammes de rayonnement de $U_{E}$ et $U_{d}$ sont pratiquement identiques jusqu'aux lobes secondaires d'ordre 2 .
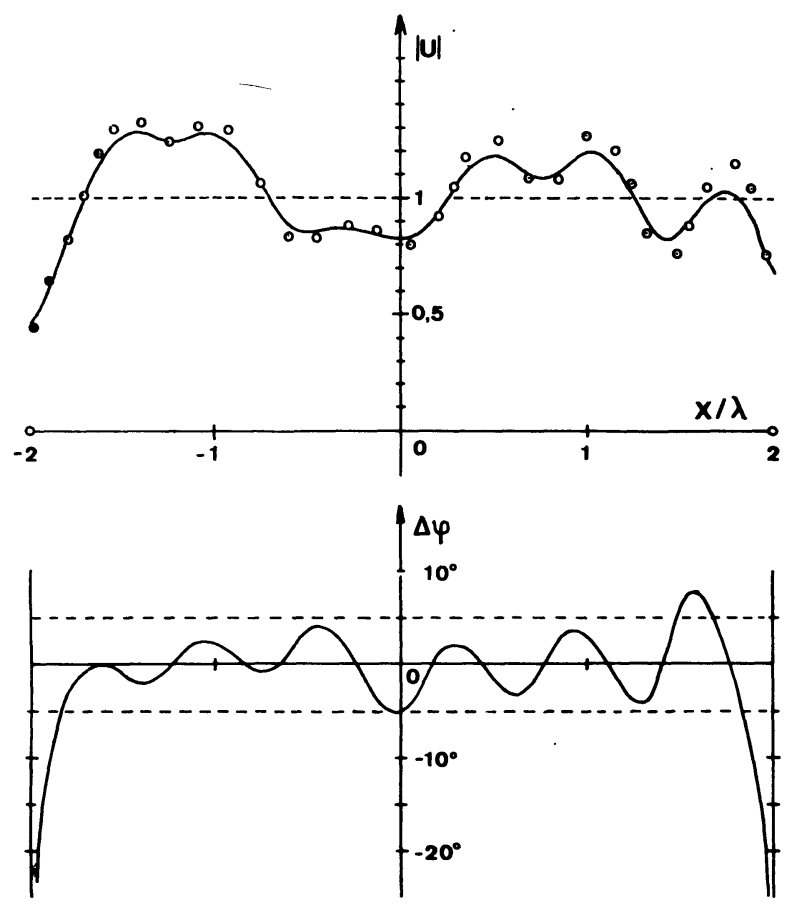

$D=4 \lambda \quad \theta=30^{\circ}$
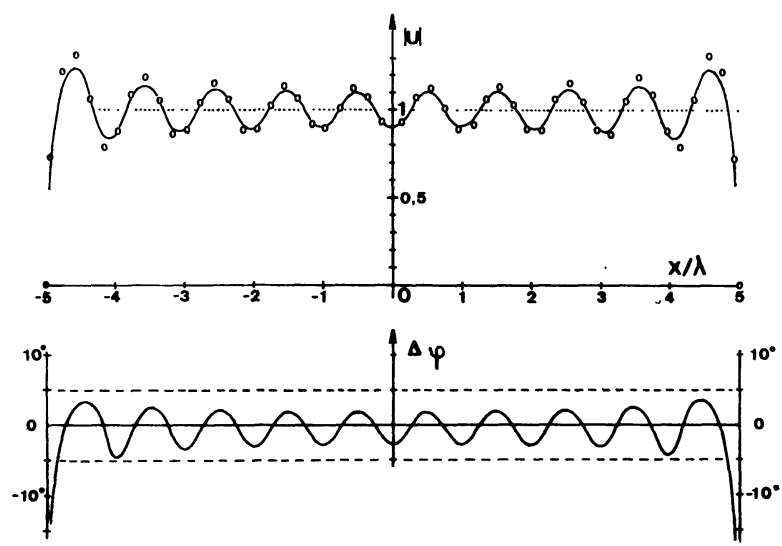

$D=10 \lambda \cdot \theta=0$
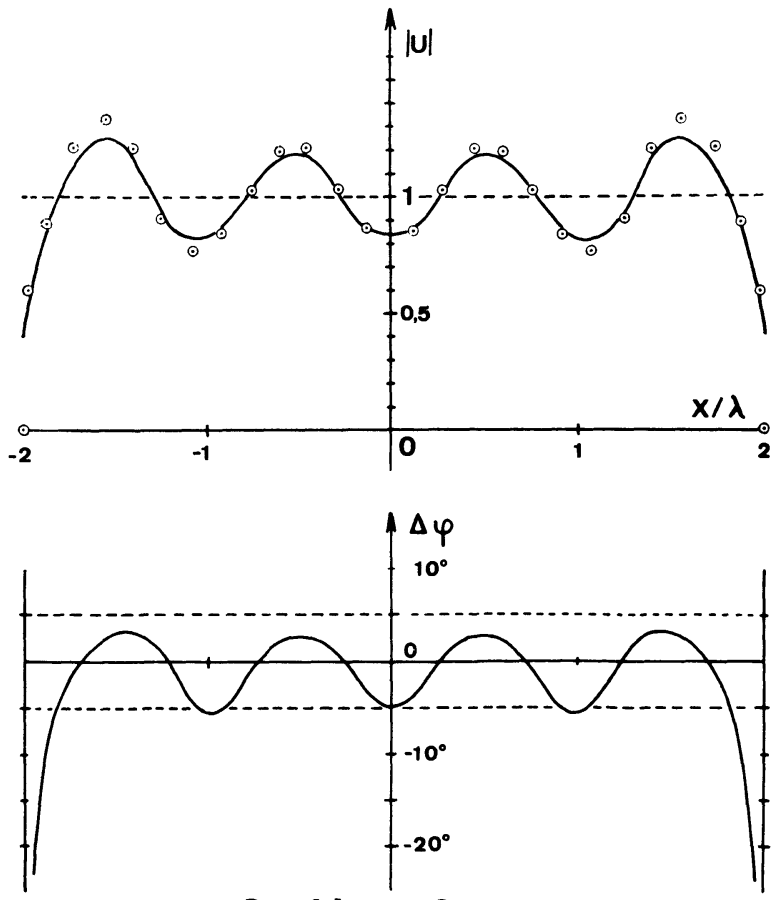

$D=4 \lambda \quad \theta=0$
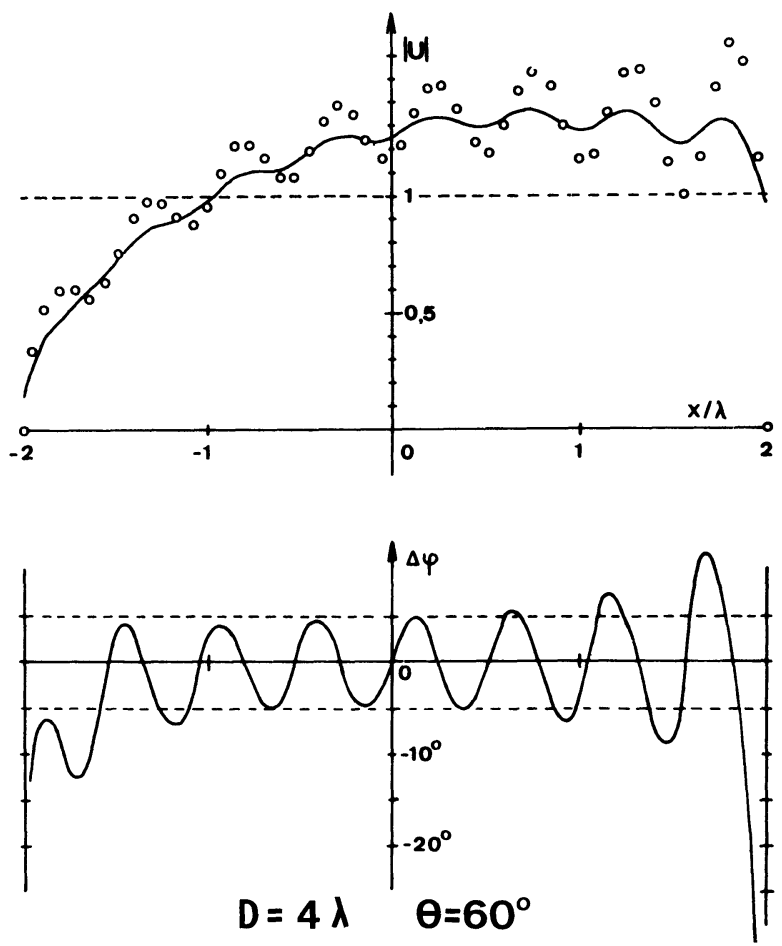

Fig. 2. - Comparaison des champs dans l'ouverture pour une fente de largeur $D$ recevant sous l'incidence $\theta$ une onde plane d'amplitude unité. Courbes $|U|=f(x / \lambda)$ : l'approximation de Rayleigh-Sommerfeld est figurée en trait plein. Le signe o correspond à la solution rigoureuse (équation intégrale (13)).

Application à un problème de diffraction. - Les propriétés des fronts d'onde quasi limités nous ont amené à les utiliser comme champ incident dans le traitement du problème de la diffraction par une lentille cylindrique. On simule ainsi la présence d'un diaphragme devant la lentille (Fig. 3). Le problème a été traité par la méthode du point matching à l'aide d'un programme de calcul mis en œuvre par W. Tabbara [7]. Pour une étude du champ dans le domaine paraxial les points d'échantillonnage et les points de calcul sont situés dans une région où l'approximation de RayleighSommerfeld donne de bons résultats. 
Nous avons, à titre d'illustration fait l'étude de $U$ dans le plan axial d'une lentille de largeur $D=4 \lambda$ et de distance focale $14,4 \lambda$ [7] illuminée sous l'incidence normale par une onde T. E. quasi limitée. Le champ incident est produit par un ruban unipolaire de

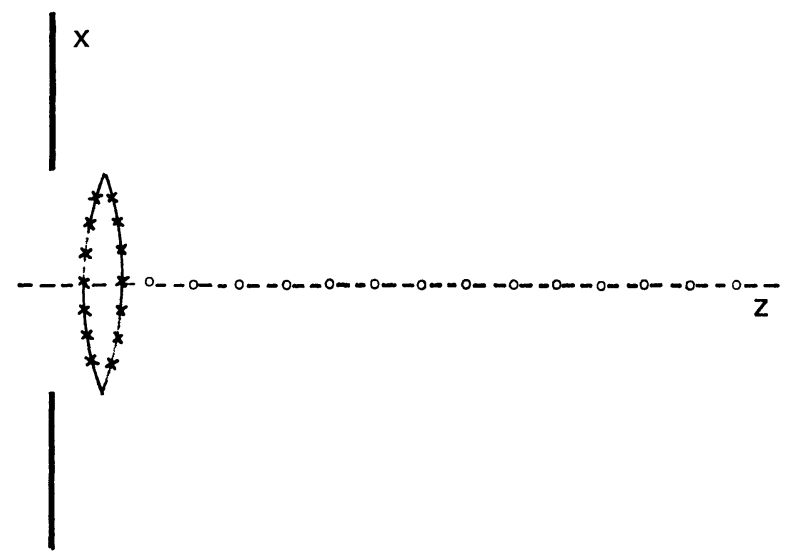

FIG. 3. - Répartition des points d'échantillonnage $(x)$ et des points de calcul (o).

largeur $4 \lambda$ situé à $0,12 \lambda$ de la première face de la lentille. L'échantillonnage utilisé comporte 32 points répartis régulièrement sur le contour de la lentille. $\mathrm{La}$ figure 4 représente dans le plan axial de la lentille, le module $|U|$ du champ électrique et l'anomalie de phase

$$
\alpha=\varphi-\varphi_{0}-k\left(z-z_{0}\right)
$$

$\varphi$ et $\varphi_{0}$ sont les phases respectives du champ aux points d'abscisses $z$ variable et $z_{0}$ fixe.
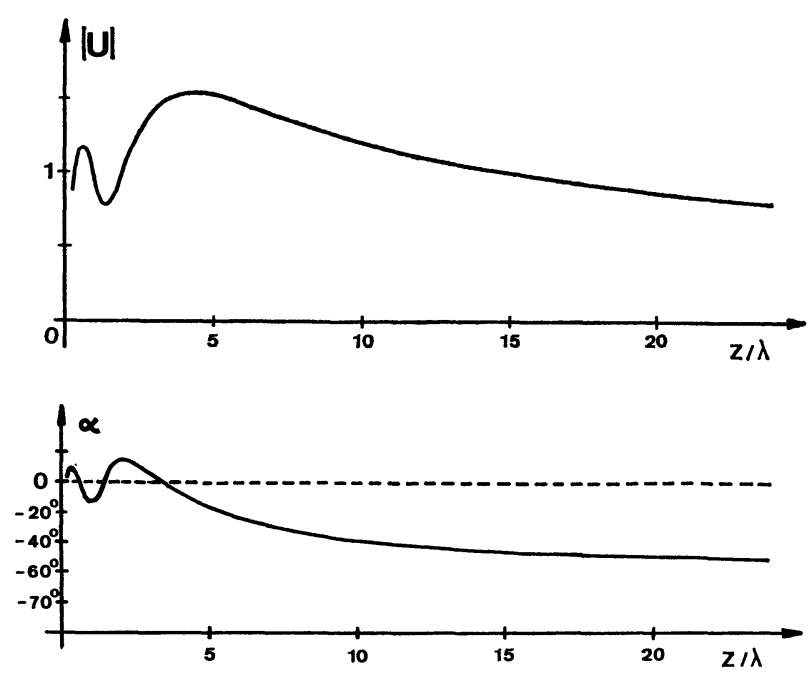

Fig. 4. - Champ et anomalie de phase dans le plan axial d'une lentille diélectrique cylindrique équiconvexe de largeur $D=4 \lambda$, de distance focale $f=14,4 \lambda$. La lentille reçoit, sous incidence normale, une onde T. E. "quasi limitée ».

\section{Bibliographie}

[1] Pavageau (J.), C. R. Acad. Sci. Paris, 1969, 268, 737.

[2] Wirgin (A.), Thèse, Université de Paris, 1967.

[3] Bouwramp (C. J.), ( Diffraction Theory ), Rep. Progr. Phys., 1954, 17, 35.

[4] FACQ (P.), C. R. Acad. Sci. Paris, 1969, 269, 853.
[5] Filippi (P.) et Dumery (G.), Acustica, 1969, 21, 343. [6] Harrington (R. F.), « Field Computation by Moment Methods ), Macmillan, New York, 1968.

[7] Tarbara (W.), Nouveau point de vue sur la formation des images par une lentille. C. R. Acad. Sci. Paris, 1970, 270B, 1092. 